\title{
Product Integration for the Generalized Abel Equation
}

\author{
By Richard Weiss
}

Abstract. The solution of the generalized Abel integral equation

$$
g(t)=\int_{0}^{t}\left\{k(t, s) /(t-s)^{\alpha}\right\} f(s) d s, \quad 0<\alpha<1,
$$

where $k(t, s)$ is continuous, by the product integration analogue of the trapezoidal method is examined. It is shown that this method has order two convergence for $\alpha \in\left[\alpha_{1}, 1\right)$ with $\alpha_{1} \div 0.2117$. This interval contains the important case $\alpha=\frac{1}{2}$. Convergence of order two for $\alpha \in\left(0, \alpha_{1}\right)$ is discussed and illustrated numerically. The possibility of constructing higher order methods is illustrated with an example.

1. Introduction. The generalized Abel integral equation

$$
g(t)=\int_{0}^{t} \frac{k(t, s)}{(t-s)^{\alpha}} f(s) d s, \quad 0<\alpha<1,0 \leqq t \leqq T<\infty,
$$

where $k(t, s)$ is continuous for $0 \leqq s \leqq t \leqq T$ and

$$
k(t, t) \neq 0 \text {, }
$$

is of considerable importance in many fields. For example, consider the equation

$$
Y(y)=2 \int_{y}^{1} \frac{R(r) r}{\left(r^{2}-y^{2}\right)^{1 / 2}} d r, \quad 0 \leqq y \leqq 1,
$$

which occurs frequently in mathematical physics. By a change of variables, (1.3) can be transformed to

$$
g(t)=\int_{0}^{t} \frac{f(s)}{(t-s)^{1 / 2}} d s, \quad 0 \leqq t \leqq 1,
$$

which is an important special case of (1.1). Various numerical methods for performing the inversion of (1.3) as well as (1.4) have been developed.-A bibliography can be found in Minerbo and Levy [1].

As a second example, consider equations of the type

$$
\begin{aligned}
(2 \pi t)^{-1 / 2} \exp [- & \left.\frac{1}{2}\{a(t)\}^{2} / t\right] \\
& =\int_{0}^{t}\left\{2 \pi(t-s)^{-1 / 2}\right\} \exp \left[-\frac{1}{2}\{a(t)-a(s)\}^{2} /(t-s)\right] f(s) d s,
\end{aligned}
$$

where $a(t)$ is a differentiable function with $a(0)>0$. Such equations and systems of

Réceived May 10, 1971.

AMS 1970 subject classifications. Primary 65L05. methods.

Key words and phrases. Generalized Abel equation, numerical solution, product integration 
similar structure appear in the analysis of diffusion processes \{see Fortet [2]\}. A method for solving (1.5) has been proposed by Durbin [3].

However, none of the methods suggested for (1.4) as well as (1.5) can be applied to the general case (1.1). Only methods based on the concept of product integration \{see Young [4]\} appear to be applicable. Such methods have been suggested on the basis of rigorous numerical experimentation by Linz [5] and Noble [6]. Independent support for this can be found in [3], though the product integration type method developed there is not generally applicable.

A theoretical justification for the use of product integration methods can be found in Weiss and Anderssen [7]. They show that the midpoint product integration method is convergent of order one if $f(s)$ and $k(t, s)$ are suitably smooth and convergent of order at least $(1-\alpha)$ if weaker smoothness conditions apply.

In this paper, we extend the result of [7] by examining the applicability of other product integration methods to (1.1). Initially, we show that the use of trapezoidal product integration leads to a method with second order convergence. The result again depends on the smoothness of $f(t)$ and $k(t, s)$. In addition, the possibility of determining higher order methods is discussed.

The existence and uniqueness of solutions of (1.1) is considered in Section 2. In Section 3, we give a basic definition and a lemma required in the subsequent analysis of Section 4, where we define the trapezoidal method and investigate its convergence. In Section 5, we examine the effect of computational errors, and a numerical example is presented in Section 6. A third order method is considered in Section 7.

2. Analytic Solution of (1.1). The existence and uniqueness of solutions of (1.1) is guaranteed under the following conditions:

THEOREM 2.1 \{KowALEWSKI [8, Section 1, pp. 80-82]\}. The integral equation (1.1) has a unique continuous solution $f(t), t \in[0, T]$, if $k(t, s)$ and $g(t)$ satisfy

(i) $k(t, s)$ and $\partial k(t, s) / \partial t$ are continuous for $0 \leqq s \leqq t \leqq T$,

(ii) $k(t, t) \neq 0$ for $t \in[0, T]$,

(iii) $G(t)=d / d t \int_{0}^{t}\left(g(s) /(t-s)^{1-\alpha}\right) d s$ is continuous for $t \in[0, T]$.

3. Preliminaries. To solve (1.1) we introduce the grid $\left\{t_{i}=i \Delta t, i=0,1, \cdots\right.$, $I=T / \Delta t\}$ with gridspacing $\Delta t$. An approximate solution, defined with respect to these grid points, will be denoted by $\tilde{f}_{i}$.

Definition 3.1. Let $\tilde{f}_{i}(\Delta t)(i=0,1, \cdots)$ denote the approximation to $f\left(t_{i}\right)$ obtained by a finite difference method with gridspacing $\Delta t$. Then, the method is said to be convergent if and only if

$$
\max _{0 \leq i \leq I}\left|\tilde{f}_{i}(\Delta t)-f\left(t_{i}\right)\right| \rightarrow 0
$$

as $\Delta t \rightarrow 0, I \rightarrow \infty$ such that $I \Delta t=T$. Furthermore, the method is said to be convergent of order $p$, if $p$ is the largest value for which there exists a finite constant $C$ and a $\Delta t_{0}$ such that

$$
\max _{0 \leq i \leq I}\left|\tilde{f}_{i}(\Delta t)-f\left(t_{i}\right)\right| \leqq C \Delta t^{D}
$$

for all $0<\Delta t \leqq \Delta t_{0}$ 
The convergence proof developed in Section 4 is based on the following lemma which is a consequence of the standard results for regular infinite systems of algebraic equations \{see Kantorovich and Krylov [9, Section 2, p. 27]\}.

LEMMA 3.1. Let the real numbers $x_{i}(i=1,2, \cdots)$ satisfy

$$
\begin{aligned}
\left|x_{1}\right| \leqq K, \quad\left|x_{2}\right| \leqq K, & K \text { is const, } \\
\left|x_{i+1}\right| \leqq \sum_{i=1}^{i}\left|a_{i, i}\right|\left|x_{i}\right|+\left|b_{i}\right| & (i=2,3, \cdots),
\end{aligned}
$$

with

$$
\begin{aligned}
\rho_{i} & =1-\sum_{i=1}^{i}\left|a_{i, j}\right|>0, \\
\left|b_{i}\right| & \leqq K \rho_{i} \quad(i=2,3, \cdots),
\end{aligned}
$$

then $\left|x_{i}\right| \leqq K(i=1,2, \cdots)$.

Throughout the paper, we use the notation

$$
g_{i}=g\left(t_{i}\right), \quad f_{i}=f\left(t_{i}\right), \quad k_{i, i}=k\left(t_{i}, t_{i}\right), \quad \epsilon_{i}=f_{i}-f_{i} .
$$

In addition, the subscripted capitals $C_{1}, C_{2}, \ldots$ will always denote positive real constants.

4. The Trapezoidal Product Integration Method. We replace $k\left(t_{i}, s\right) f(s)$ in

$$
g_{i}=\int_{0}^{t_{i}} \frac{k\left(t_{i}, s\right)}{\left(t_{i}-s\right)^{\alpha}} f(s) d s \quad(i=1, \cdots, I)
$$

by the piecewise linear function

$$
\begin{aligned}
M_{\Delta t}\left(t_{i}, s\right)=\left[\left(t_{i+1}-s\right) k_{i, j} \tilde{f}_{i}+\left(s-t_{i}\right) k_{i, j+1} \tilde{f}_{i+1}\right] / \Delta t \\
\\
\quad\left\{t_{i} \leqq s \leqq t_{i+1}(j=0, \cdots, i-1)\right\},
\end{aligned}
$$

and thus obtain the following lower triangular system for $\tilde{f}_{i}(i=1, \cdots, I)$ :

$$
\sum_{i=1}^{i} \tilde{f}_{i} k_{i, j} W_{i-i}=g_{i} /\left(\Delta t^{1-\alpha} \phi(\alpha)\right)-\tilde{f}_{0} k_{i, 0} W_{i} \quad(i=1, \therefore, n,
$$

where

$$
\begin{gathered}
\phi(\alpha)=\frac{1}{1-\alpha}-\frac{1}{2-\alpha}, \\
W_{0}=\frac{1}{\phi(\alpha) \Delta t^{2-\alpha}} \int_{0}^{\Delta t} \frac{\Delta t-t}{t^{\alpha}} d t=1, \\
W_{l}=\frac{1}{\phi(\alpha) \Delta t^{2-\alpha}}\left(\int_{(l-1) \Delta t}^{l \Delta t} \frac{t-(l-1) \Delta t}{t^{\alpha}} d t+\int_{l \Delta t}^{(l+1) \Delta t} \frac{(l+1) \Delta t-t}{t^{\alpha}} d t\right) \\
=(l+1)^{2-\alpha}-2 l^{2-\alpha}+(l-1)^{2-\alpha} \quad(l=1, \cdots, I-1),
\end{gathered}
$$




$$
\begin{aligned}
W_{l} & =\frac{1}{\phi(\alpha) \Delta t^{2-\alpha}} \int_{(l-1) \Delta t}^{l \Delta t} \frac{t-(l-1) \Delta t}{t^{\alpha}} d t \\
& =\frac{1}{\phi(\alpha)}\left\{\frac{1}{2}-\frac{1}{\alpha}\left(l^{2-\alpha}-(l-1)^{2-\alpha}\right)-\frac{(l-1)}{1-\alpha}\left(l^{1-\alpha}-(l-1)^{1-\alpha}\right)\right\} \\
& (l=1, \cdots, l) .
\end{aligned}
$$

For the starting value $\tilde{f}_{0}$, we take

$$
f_{0}=f_{0}=\lim _{t \rightarrow 0}\left(g(t) t^{\alpha-1}\right)(1-\alpha) / k_{0,0}
$$

which exists, whenever (1.1) has a continuous solution. It follows from (1.2) that (4.1) is nonsingular.

The numerical process (4.1) defines the product integration analogue of the trapezoidal rule applied to nonsingular first kind Volterra equations \{see Linz [10]\}. We shall refer to it as the trapezoidal product integration method.

As in [7], we first investigate the convergence of (4.1) for the case $k(t, s) \equiv 1$.

THEOREM 4.1. If $f(t)$ is three times differentiable and $f^{\prime \prime \prime}(t)$ is bounded for $0 \leqq t \leqq T$, then, for $\alpha \in\left[\alpha_{1}, 1\right)$ with $\alpha_{1} \doteqdot 0.2117,(4.1)$ is convergent of order 2 .

Let $f_{\Delta}(s)$ denote the piecewise linear function interpolating $f(s)$ at the grid points, $f_{\Delta t}(s)=\left[\left(t_{i+1}-s\right) f_{i}+\left(s-t_{i}\right) f_{i+1}\right] / \Delta t \quad\left\{t_{i} \leqq s \leqq t_{i+1}(j=0, \cdots, I-1)\right\}$, and let

$$
e_{\Delta t}(s)=f_{\Delta t}(s)-f(s)
$$

By subtracting

$$
\frac{1}{\Delta t^{1-\alpha} \phi(\alpha)}\left(\int_{0}^{t_{i}} \frac{f_{\Delta t}(s)}{\left(t_{i}-s\right)^{\alpha}} d s-\int_{0}^{t_{i}} \frac{e_{\Delta t}(s)}{\left(t_{i}-s\right)^{\alpha}}-g_{i}\right)=0
$$

from (4.1), we obtain

$$
\sum_{i=1}^{i} \epsilon_{i} W_{i-j}=-\frac{1}{\Delta t^{1-\alpha} \phi(\alpha)} \int_{0}^{t^{\prime}} \frac{e_{\Delta t}(s)}{\left(t_{i}-s\right)^{\alpha}} d s-\epsilon_{0} W_{i} \quad(i=1, \cdots, I) .
$$

Finally, subtraction of (4.2) from (4.2), with $i$ replaced by $i+1$, yields the basic error equation

$$
\epsilon_{i+1}=\sum_{i=1}^{i} \epsilon_{i} a_{i-i}+b_{i} \quad(i=1, \cdots, I-1),
$$

with

$$
\begin{gathered}
a_{0}=W_{0}-W_{1}=3-2^{2-\alpha}, \quad a_{l}=W_{l}-W_{l+1} \quad(l=1, \cdots, I-1), \\
b_{i}=-\frac{1}{\phi(\alpha) \Delta t^{1-\alpha}} \beta_{i}^{1}+\beta_{i}^{2},
\end{gathered}
$$

$$
\begin{gathered}
\beta_{i}^{1}=\int_{t_{i}}^{t_{i+1}} \frac{e_{\Delta t}(s)}{\left(t_{i+1}-s\right)^{\alpha}} d s-\sum_{i=0}^{i-1} \int_{t_{i}}^{t_{i+1}}\left(\frac{1}{\left(t_{i}-s\right)^{\alpha}}-\frac{1}{\left(t_{i+1}-s\right)^{\alpha}}\right) e_{\Delta t}(s) d s, \\
\beta_{i}^{2}=\epsilon_{0}\left(W_{i}-W_{i+1}\right) .
\end{gathered}
$$


Before we give a proof of the theorem, it is first necessary to obtain some preliminary results.

LEMMA 4.1.

$$
\begin{array}{ll}
\left|\beta_{i}^{1}\right| \leqq C_{1} \Delta t^{3-\alpha} i^{-\alpha} & (i=1, \cdots, I-1), \\
\left|\beta_{i}^{2}\right| \leqq C_{2} \Delta t^{2} i^{-1-\alpha} & (i=1, \cdots, I-1), \text { if }\left|\epsilon_{0}\right| \leqq C_{3} \Delta t^{2} .
\end{array}
$$

Proof. By a standard theorem on Lagrange interpolation

$$
\begin{aligned}
e_{\Delta t}(s)=\frac{1}{2}\left(s-t_{i}\right)\left(t_{i+1}-s\right) f^{\prime \prime}\left(t_{i}\right)+ & r_{i}(s) \\
& \left\{t_{i} \leqq s \leqq t_{i+1}(j=0, \cdots, I-1)\right\} .
\end{aligned}
$$

with

$$
\left|r_{i}(s)\right| \leqq \frac{1}{8} F_{3} \Delta t^{8}, \quad F_{3}=\max _{t \in[0, T]} f^{\prime \prime \prime}(t) .
$$

By substituting (4.5) into (4.4), we obtain

$$
\beta_{i}^{1}=\Delta t^{8-\alpha}\left(f^{\prime \prime}\left(t_{i}\right) \gamma-\sum_{i=0}^{i-1} f^{\prime \prime}\left(t_{i}\right) \theta_{i-i-1}\right)+\delta_{i}
$$

where

$$
\begin{aligned}
& \gamma=\frac{1}{2 \Delta t^{3-\alpha}} \int_{0}^{\Delta t} s^{1-\alpha}(\Delta t-s) d s, \\
& \theta_{l}=\frac{1}{2 \Delta t^{3-\alpha}} \int_{0}^{\Delta t} s(\Delta t-s)\left(\frac{1}{(l \Delta t+s)^{\alpha}}-\frac{1}{((l+1) \Delta t+s)^{\alpha}}\right) d s, \\
& \delta_{i}=\int_{t,}^{t_{i+1}} \frac{r_{i}(s)}{\left(t_{i+1}-s\right)^{\alpha}} d s-\sum_{i=0}^{i-1} \int_{t_{1}}^{t_{i+1}}\left(\frac{1}{\left(t_{i}-s\right)^{\alpha}}-\frac{1}{\left(t_{i+1}-s\right)^{\alpha}}\right) r_{i}(s) d s .
\end{aligned}
$$

It can easily be verified that, by (4.6),

$$
\left|\delta_{i}\right| \leqq C_{4} \Delta t^{4-\alpha} \quad(i=1, \cdots, I-1),
$$

and that

$$
\begin{aligned}
0<\theta_{0}, 0<\theta_{l}<C_{5} I^{1-\alpha}, & l \geqq 1, \\
\left|\gamma-\sum_{i=0}^{i-1} \theta_{l}\right| \leqq C_{6} i^{-\alpha}, & i \geqq 1 .
\end{aligned}
$$

By partial summation,

$$
\begin{aligned}
& \sum_{i=0}^{i-1} f^{\prime \prime}\left(t_{i}\right) \theta_{i-i-1}=f^{\prime \prime}\left(t_{i-1}\right) \sum_{i=0}^{i-1} \theta_{l}+\sum_{\nu=1}^{i-1}\left(f^{\prime \prime}\left(t_{i-\nu-1}\right)-f^{\prime \prime}\left(t_{i-\nu}\right)\right) \times \sum_{i=\nu}^{i-1} \theta_{l} \\
& (i=2, \cdots, I-1)
\end{aligned}
$$

and, using (4.8),

(4.10) $\left|\sum_{\nu=1}^{i-1}\left(f^{\prime \prime}\left(t_{i-\nu-1}\right)-f^{\prime \prime}\left(t_{i-\nu}\right)\right) \times \sum_{i=-}^{i-1} \theta_{l}\right| \leqq C_{7} \Delta t i^{1-\alpha} \quad(i=2, \cdots, I-1)$. 
Combining (4.9) and (4.10), we obtain

$$
\begin{aligned}
& \left|f^{\prime \prime}\left(t_{i}\right) \gamma-\sum_{i=0}^{i-1} f^{\prime \prime}\left(t_{i}\right) \theta_{i-j-1}\right| \\
& \leqq\left|f^{\prime \prime}\left(t_{i}\right)\left(\gamma-\sum_{i=0}^{i-1} \theta_{l}\right)\right|+\left|f^{\prime \prime}\left(t_{i}\right)-f^{\prime \prime}\left(t_{i-1}\right)\right| \times \sum_{i=0}^{i-1} \theta_{l}+C_{7} \Delta t i^{1-\alpha} \\
& \leqq F_{2} C_{8} i^{-\alpha}+\gamma F_{8} \Delta t+C_{7} \Delta t i^{1-\alpha} \\
& (i=1, \cdots, I-1), F_{2}=\max _{t \in[0, T 1} f^{\prime \prime}(t) .
\end{aligned}
$$

Since

$$
\Delta t=T / I \leqq T / i \quad(i=1, \cdots, I),
$$

it follows from (4.7) and (4.11) that

$$
\left|\beta_{i}^{1}\right| \leqq C_{1} \Delta t^{3-\alpha} i^{-\alpha} \quad(i=1, \cdots, I-1) .
$$

The second inequality of Lemma 4.1 is an immediate consequence of the asymptotic expansion of $\left(\bar{W}_{i}-\bar{W}_{i+1}\right)$. $\square$

Proof of Theorem 4.1. Using Lemma 4.1 it is clear that

$$
\left|b_{i}\right| \leqq C_{8} \Delta t^{2} i^{-\alpha} \quad(i=1, \cdots, I-1) .
$$

Returning to (4.3), we note that

$$
\begin{aligned}
\sum_{l=0}^{i-1} a_{l} & =1-W_{i}, \quad l \geqq 1, \\
a_{l} & >0, \quad l \geqq 1, \\
0 \leqq a_{0} & <1, \quad \alpha \in\left[\alpha_{0}, 1\right),
\end{aligned}
$$

$$
-1<a_{0}<0, \quad \alpha \in\left(0, \alpha_{0}\right), \quad \alpha_{0}=2-\frac{\ln 3}{\ln 2} \div 0.4150 .
$$

Substituting for $\epsilon_{i}$ in (4.3), we obtain

$$
\epsilon_{i+1}=\sum_{i=1}^{i} \epsilon_{i} a_{i-i}^{1}+b_{i}+a_{0} b_{i-1} \quad(i=2, \cdots, I-1),
$$

with

$$
a_{0}^{1}=0, \quad a_{l}^{1}=a_{l}+a_{0} a_{l-1}, \quad l \geqq 1,
$$

and

$$
\begin{array}{ll}
a_{1}^{1}>0, & \alpha \in(0,1), \\
a_{l}^{1} \geqq 0, \quad l \geqq 2, & \alpha \in\left[\alpha_{1}, 1\right), \alpha_{1} \doteqdot 0.2117, \\
a_{2}^{1}<0, & \alpha \in\left(0, \alpha_{1}\right) .
\end{array}
$$

The latter is a consequence of the structure of $\left\{a_{l}(\alpha), l \geqq 0\right\}$. From (4.13),

$$
\sum_{i=0}^{i-1} a_{l}^{1}=1-\left(W_{i}+a_{0} W_{i-1}\right), \quad i \geqq 2,
$$


and, since

$$
W_{i}=a_{i}+W_{i+1}=\sum_{\nu=i}^{\infty} a_{\nu}, \quad i \geqq 0,
$$

it follows from (4.17) that

$$
W_{i}+a_{0} W_{i-1}=\sum_{\nu=i}^{\infty}\left(a_{\nu}+a_{0} a_{\nu-1}\right)=\sum_{\nu=i}^{\infty} a_{\nu}^{1}>0, \quad i \geqq 2, \alpha \in\left(\alpha_{1}, 1\right) .
$$

Consequently,

$$
\sum_{i=0}^{i-1} a_{l}^{1} \leqq 1-C_{9} i^{-\alpha} \quad(i=2, \cdots, I-1), \alpha \in\left[\alpha_{1}, 1\right),
$$

since $W_{i}$ behaves asymptotically like $i^{-\alpha}$.

It follows from (4.2) that

$$
\left|\epsilon_{1}\right| \leqq C_{10} \Delta t^{2}, \quad\left|\epsilon_{2}\right| \leqq C_{10} \Delta t^{2} .
$$

We are now in a position to apply Lemma 3.1 to (4.16). On the basis of (4.19) together with (4.17), (4.18) and (4.12), the conditions of the lemma are satisfied and the required result

$$
\left|\epsilon_{i}\right| \leqq C_{11} \Delta t^{2} \quad(i=0, \cdots, I), \alpha \in\left[\alpha_{1}, 1\right)
$$

follows.

Note 4.1. Using Lemma 3.1 and (4.13)-(4.15), we can prove second order convergence for (4.1) with $\alpha \in\left[\alpha_{0}, 1\right)$ without making a substitution of the type \{see (4.16) \} introduced in the proof of Theorem 4.1. On the other hand, if we substitute for $\epsilon_{i-2}$ instead of $\epsilon_{i}$ in (4.16) using (4.3), we have

$$
\epsilon_{i+1}=\sum_{i=1}^{i} \epsilon_{i} a_{i-i}^{2}+b_{i}+a_{0} b_{i-1}+a_{2}^{1} b_{i-3} \quad(i=4, \cdots, I-1)
$$

with

$$
a_{0}^{2}=0, \quad a_{1}^{2}=a_{1}^{1}, \quad a_{2}^{2}=0, \quad a_{l}^{2}=a_{l}^{1}+a_{2}^{1} a_{l-3}, \quad l \geqq 3 .
$$

It can be shown numerically that (4.20) satisfies the conditions of Lemma 3.1 for $\alpha \in\left[\alpha_{2}, 1\right)$ with $\alpha_{2} \doteqdot 0.1292$. It follows that, if the proof of order 2 convergence for all $\alpha, 0<\alpha<1$, by means of Lemma 3.1 is possible, it will involve a complex inductive argument. However, because our proof already covers the case $\alpha=\frac{1}{2}$, which is the most important one in application, an extension of the result of Theorem 4.1 will not be pursued further in this paper. Numerical experimentation indicates that second order convergence holds for all $\alpha \in(0,1)$. This is illustrated in Section 6 .

Since the extension of Theorem 4.1 to a general $k(t, s)$ can be established using a technique analogous to that of [7] for midpoint product integration, we only state the result.

THEOREM 4.2. If

(i) $f(t)$ satisfies the conditions of Theorem 4.1,

(ii) $k(t, s)$ is twice continuously differentiable with respect to $t$ and $s$ for $0 \leqq s \leqq t \leqq T$, and 
(iii) $\partial^{3} k(t, s) / \partial s^{3}$ and $\partial^{3} k(t, s) / \partial t \partial s^{2}$ are bounded for $0 \leqq s \leqq t \leqq T$, then, for $\alpha \in\left[\alpha_{1}, 1\right),(4.1)$ is convergent of order 2 .

5. Computational Errors. During the actual application of algorithm (4.1), the values $\left\{\bar{f}_{0}, \cdots, \bar{f}_{I}\right\}$ instead of $\left\{\tilde{f}_{0}, \cdots, \bar{f}_{I}\right\}$, which satisfy $\bar{f}_{0}=f_{0}+\eta_{0}$,

$$
\sum_{i=1}^{i} \bar{f}_{i} k_{i, j} W_{i-i}=g_{i} /\left(\Delta t^{1-\alpha} \phi(\alpha)\right)-\bar{f}_{0} k_{i, 0} W_{i}+\eta_{i} \quad(i=1, \cdots, n,
$$

are determined, where the perturbations $\left\{\eta_{i}, i=0, \cdots, I\right\}$ represent the effect of rounding and truncation errors. It follows from Lemma 3.1 that

$$
\left|\tilde{f}_{i}-\bar{f}_{i}\right| \leqq K_{1} \eta\left(1+i^{\alpha}\right) \quad(i=0, \cdots, n), \alpha \in[\alpha, 1),
$$

where $K_{1}=$ const $>0$ and $\left|\eta_{i}\right| \leqq \eta(i=0, \cdots, I)$. However, since the application of Lemma 3.1 cannot yield a sharp estimate in this case, (5.1) represents a conservative bound. Numerical experimentation indicates that

$$
\max _{0 \leq i \leq I}\left|\tilde{f}_{i}-\bar{f}_{i}\right| \leqq K_{2} \eta \text {, }
$$

where $K_{2}$ is a positive constant depending on $T$. This is the usual behaviour associated with computational errors in the numerical solution of Volterra integral equations of the second kind; equations of the first kind tend to be less well conditioned.

6. A Numerical Example. The trapezoidal product integration method was applied to the following equation:

$$
\frac{t^{1-\alpha}}{(1-\alpha) 2 i}\left[F_{1}(1 ; 2-\alpha ; i t)-{ }_{1} F_{1}(1 ; 2-\alpha ;-i t)\right]=\int_{0}^{t} \frac{f(s)}{(t-s)^{\alpha}} d s,
$$

$$
0 \leqq t \leqq \pi,
$$

where ${ }_{1} F_{1}$ is Kummer's hypergeometric function \{see Erdélyi [11, Section 13, p. $189]\}$. ( $i$ is the imaginary unit.)

The solution is $f(t)=\sin t$.

The errors obtained for $\alpha=0.5$, for which convergence is guaranteed by Theorem 4.1, and for $\alpha=0.05$ are listed in Table 6.1. In both cases, the error satisfies the predicted $\Delta t^{2}$ dependence.

Remark. All numerical results presented in this paper were computed in double precision arithmetic on the IBM 360/50 computer at the Australian National University.

7. A Third Order Method. The existence of higher order methods for (1.1) is not immediately obvious. So far, convergent methods of order greater than 2 have not been found for the first kind Volterra equation

$$
g(t)=\int_{0}^{t} k(t, s) f(s) d s,
$$

where $k(t, s)$ satisfies (1.2) \{see [10]\}. Equation (7.1) can be considered as a special case of (1.1) with $\alpha=0$. Linz [5] has shown numerically that fourth order methods can be constructed for the equation 


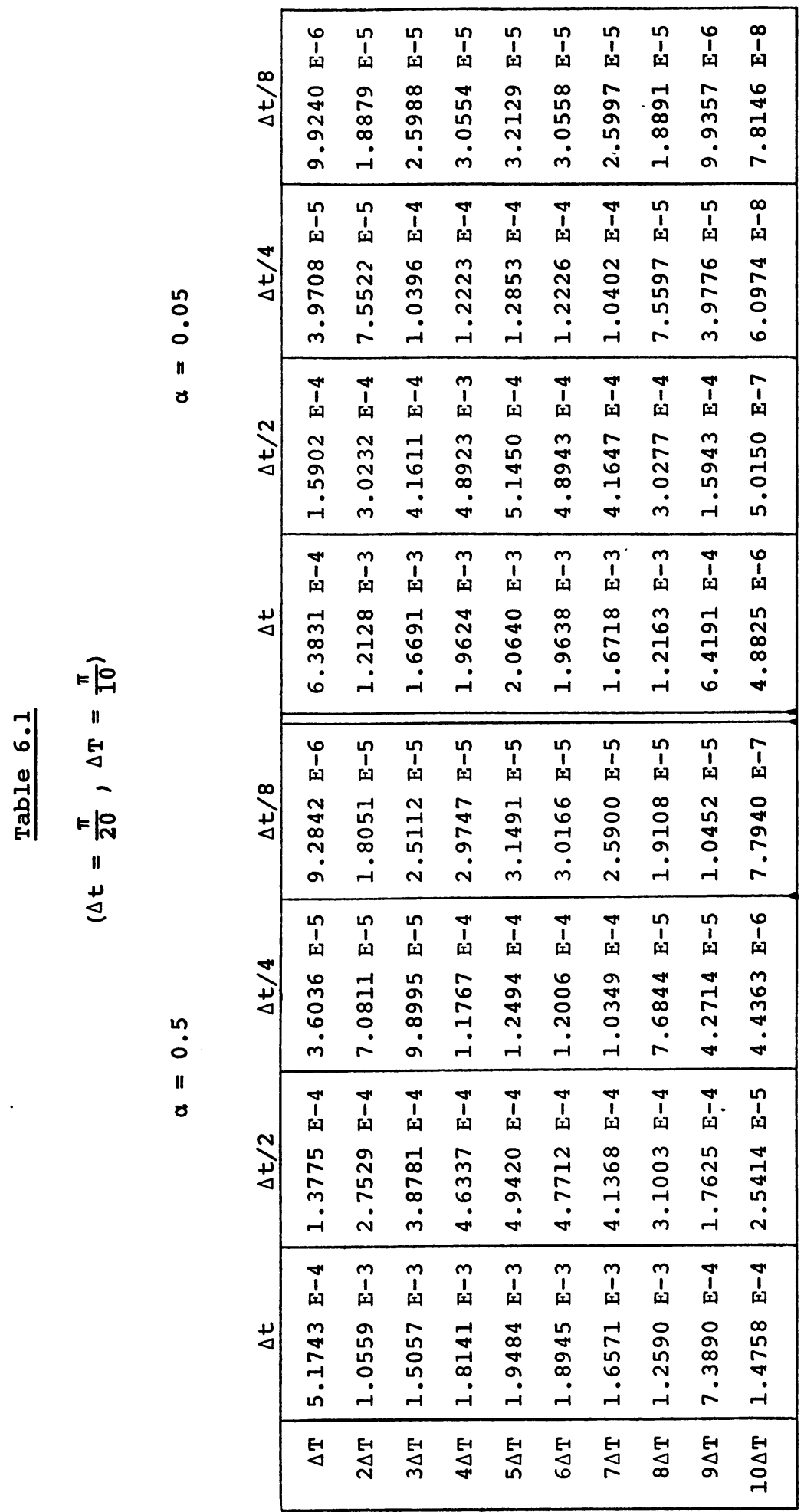




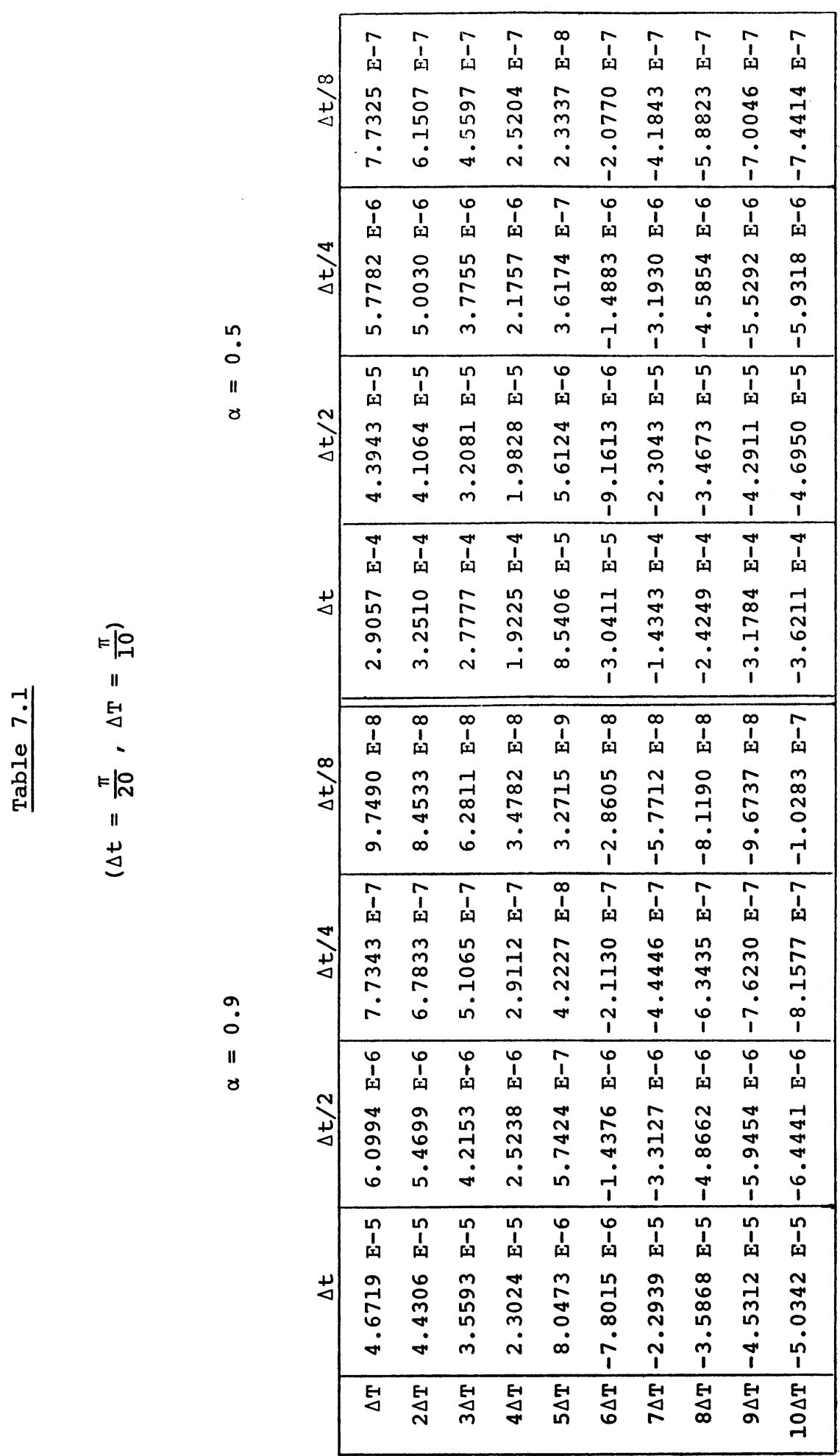




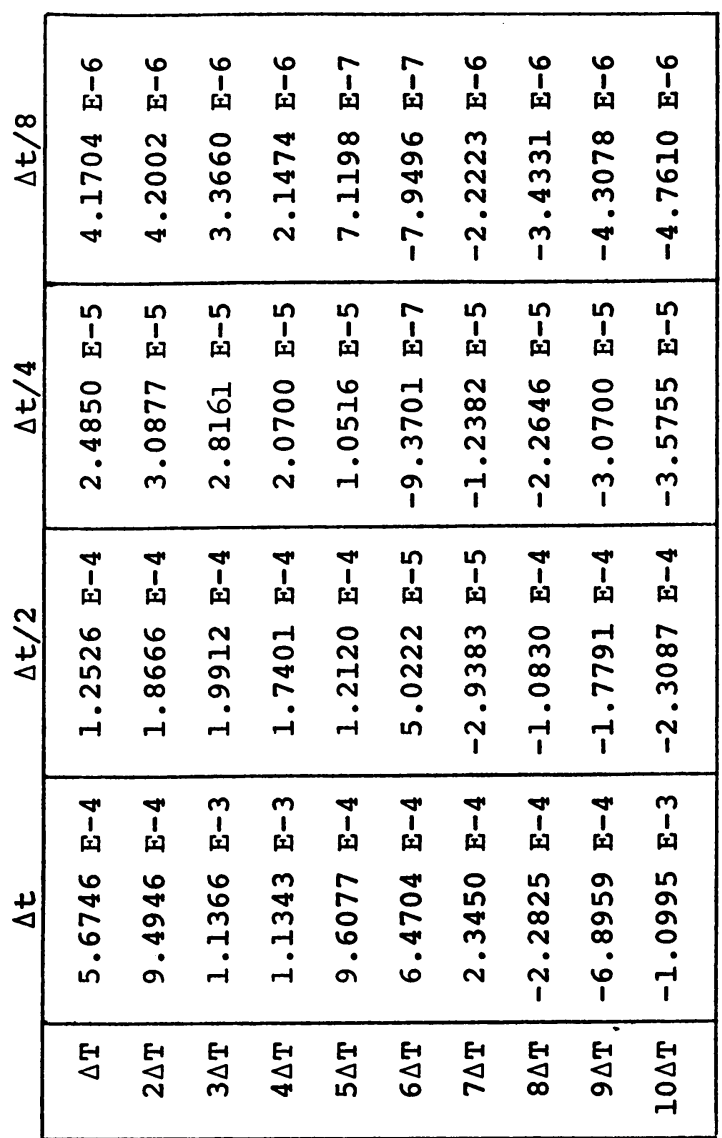




$$
g(t)=\int_{0}^{t} \frac{f(s)}{\left(t^{2}-s^{2}\right)^{1 / 2}} d s, \quad 0 \leqq t \leqq T<\infty .
$$

However, it is not possible to infer the existence of higher order methods for (1.1) on the basis of this existence, since the integral operator

$$
A f(t)=\int_{0}^{t} \frac{f(s)}{(t-s)^{\alpha}} d s, \quad \alpha<1,
$$

is a compact operator from $C[0, T]$ to $C[0, T]$, while the operator

$$
B f(t)=\int_{0}^{t} \frac{f(s)}{\left(t^{2}-s^{2}\right)^{1 / 2}} d s
$$

is not.

We verify numerically the existence of higher order methods for (1.1). In fact, we construct a third order one. For simplicity, we take $k(t, s) \equiv 1$. The generalization is straightforward. We assume $I=T \Delta t$ is even and, generalizing the technique used in the development of the trapezoidal method, we approximate $f(s)$ by a function $M_{\Delta t}(s)$ which is a quadratic polynomial in each of the intervals $\left[t_{2 j}, t_{2 j+2}\right](j=0, \cdots$, $I / 2-1)$ :

$$
\begin{array}{r}
M_{\Delta t}(s)=\tilde{f}_{2 i}+\frac{s-t_{2 i}}{\Delta t}\left(\tilde{f}_{2 i+1}-\tilde{f}_{2 i}\right)+\frac{\left(s-t_{2 i}\right)\left(s-t_{2 i+1}\right)}{2 \Delta t^{2}}\left(\tilde{f}_{2 i+2}-2 \tilde{f}_{2 j+1}+\tilde{f}_{2 i}\right) \\
\left\{t_{2 i} \leqq s \leqq t_{2 i+2}(j=0, \cdots, I / 2-1)\right\} .
\end{array}
$$

By substituting $M_{\Delta t}(s)$ for $f(s)$ in (1.1), we obtain

$$
\begin{aligned}
& g_{2 i+1}=\int_{0}^{t, j+1} \frac{M_{\Delta t}(s)}{\left(t_{2 j+1}-s\right)^{\alpha}} d s \quad(j=0, \cdots, I / 2-1) \\
& g_{2 i+2}=\int_{0}^{t, s+3} \frac{M_{\Delta t}(s)}{\left(t_{2 j+2}-s\right)^{\alpha}} d s
\end{aligned}
$$

which, for each $j$, is a nonsingular two by two system for $f_{2 j+1}$ and $f_{2 i+2}$. Thus, (7.2) is a block by block method in the sense of [10]. Evaluation of the integrals in (7.2) and division by $\Delta t^{1-\alpha}$ yields

$$
\begin{gathered}
E_{-1} \tilde{f}_{2}+F_{-1} \tilde{f}_{1}+G_{-1} \tilde{f}_{0}=g_{1} / \Delta t^{1-\alpha}, \\
E_{0} \tilde{f}_{2}+F_{0} \tilde{f}_{1}+G_{0} \tilde{f}_{0}=g_{2} / \Delta t^{1-\alpha}, \\
E_{-1} \tilde{f}_{2 i+2}+F_{-1} \tilde{f}_{2 i+1}+\sum_{i=1}^{2 i} \tilde{f}_{j} V_{2 i+1-i}+\tilde{f}_{0} G_{2 i-1}=g_{2 i+1} / \Delta t^{1-\alpha} \\
\quad(i=1, \cdots, I / 2-1), \\
E_{0} \tilde{f}_{2 i+2}+F_{0} \tilde{f}_{2 i+1}+\sum_{i=1}^{2 i} \tilde{f}_{i} W_{2 i+1-i}+\tilde{f}_{0} G_{2 i}=g_{2 i+2} / \Delta t^{1-\alpha} \\
\tilde{f}_{0}=\lim _{t \rightarrow 0}\left(g(t) t^{\alpha-1}\right)(1-\alpha), \quad(l \geqq 0) ; \\
V_{2 l}=F_{2 l-1} \quad(l \geqq 1) ; \quad V_{2 l+1}=G_{2 l-1}+E_{2 l+1} \quad(l \geqq 0
\end{gathered}
$$




$$
\begin{array}{rlrl}
W_{2 l} & =F_{2 l} \quad(l \geqq 1) ; \quad W_{2 l+1}=G_{2 l}+E_{2 l+2} \quad(l \geqq 0) ; \\
E_{-1} & =\frac{1}{2}\left(\frac{1}{3-\alpha}-\frac{1}{2-\alpha}\right), \quad F_{-1}=\left(\frac{1}{1-\alpha}-\frac{1}{3-\alpha}\right), & \\
G_{-1}=\frac{1}{2}\left(\frac{1}{3-\alpha}+\frac{1}{2-\alpha}\right) ; \\
E_{i}=L_{1}(j, \alpha)\left(1+\frac{3}{2} j+\frac{1}{2} j^{2}\right)-L_{2}(j, \alpha)\left(\frac{3}{2}+j\right)+\frac{1}{2} L_{3}(j, \alpha) & (j \geqq 0) ; \\
F_{i} & =-L_{1}(j, \alpha)\left(2 j+j^{2}\right)+L_{2}(j, \alpha)(2+2 j)-L_{3}(j, \alpha) & (j \geqq 0) ; \\
G_{i} & =L_{1}(j, \alpha)\left(\frac{1}{2} j+\frac{1}{2} j^{2}\right)-L_{2}(j, \alpha)\left(\frac{1}{2}+j\right)+\frac{1}{2} L_{3}(j, \alpha) & (j \geqq 0) ; \\
L_{1}(j, \alpha) & =\left((j+2)^{1-\alpha}-j^{1-\alpha}\right) /(1-\alpha) ; \\
L_{2}(j, \alpha) & =\left((j+2)^{2-\alpha}-j^{2-\alpha}\right) /(2-\alpha) ; \\
L_{3}(j, \alpha) & =\left((j+2)^{3-\alpha}-j^{3-\alpha}\right) /(3-\alpha) .
\end{array}
$$

This method was applied to (6.1). The errors for the cases $\alpha=0.9, \alpha=0.5$ and $\alpha=0.1$ are tabulated in Table 7.1. Third order convergence holds for $\alpha=0.9$ and $\alpha=0.5$. For $\alpha=0.1$, a strict $\Delta t^{3}$ dependence is no longer apparent. However, the process is still convergent-no explosion in the errors is observed. The numerical approximations oscillate about the true solution, and the oscillations increase as $\alpha$ tends to zero. The simplest interpretation that can apply to such behaviour is one of increasing potential instability as $\alpha$ decreases.

A rigorous analysis of the convergence properties of this method cannot be given here. The problem is the lack of accurate estimates for the solution of the lower triangular system of equations which determines the behaviour of the error.

Acknowledgment. The author wishes to thank Dr. R. S. Anderssen for his assistance and helpful comments during the preparation of this paper.

The Australian National University

Computer Centre

Canberra, A.C.T. 2601, Australia

1. Gerald N. Minerbo \& Maurice E. LeVy, "Inversion of Abel's integral equation by means of orthogonal polynomials," SIAM J. Numer. Anal., v. 6, 1969, pp. 598-616. MR 41 \#6426.

2. R. M. Fortet, "Les fonctions aléatoires du type de Markoff associées à certaines équations linéaires aux derivées partielles du type parabolique," J. Math. Pures Appl., v. 22, 1943, pp. 177-243. MR 7, 19.

3. J. DuRbin, "Boundary-crossing probabilities for the Brownian motion and Poisson processes and techniques for computing the power of the Kolmogorov-Smirnov test," J. Appl. Probability (To appear.)

4. A. YouNG, "The application of approximate product-integration to the numerical solution of integral equations," Proc. Roy. Soc. London Ser. A, v. 224, 1954, pp. 561-573. MR 16, 179.

5. P. LINz, Applications of Abel Transforms to the Numerical Solution of Problems in Electrostatics and Elasticity, MRC Technical Summary Report \#826, University of Wisconsin, Madison, Wis., 1967.

6. B. NOBLE, Lecture Notes, 1970.

7. R. WEISS \& R. S. ANDERSSEN, "A product integration method for a class of singular first kind Volterra equations," Numer. Math. (To appear.)

8. G. KowalewskI, Integralgleichungen, de Gruyter, Berlin und Leipzig, 1930. 
9. L. V. Kantorovič \& V. I. KRYLOV, Approximate Methods of Higher Analysis, 3rd ed., GITTL, Moscow, 1950; English transl., Interscience, New York; Noordhoff, Groningen, 1958. MR 13, 77; MR 21 \#5268.

10. P. LnNz, The numerical solution of Volterra integral equations by finite difference methods, MRC Technical Summary Report \#825, University of Wisconsin, Madison, Wis., 1967.

11. A. ERdélyI, ET al., Tables of Integral Transforms. Vol. II, McGraw-Hill, New York, 1954. MR 16, 468. 